\title{
NONLINEAR IMPULSIVE VOLTERRA INTEGRAL EQUATIONS IN BANACH SPACES AND APPLICATIONS ${ }^{1}$
}

\author{
DAJUN GUO ${ }^{2}$ \\ Shangdong University, Jinan \\ Department of Mathematics \\ Shangdong 250100, PEOPLE'S REPUBLIC OF CHINA
}

\begin{abstract}
In this paper, we first extend results on the existence of maximal solutions for nonlinear Volterra integral equations in Banach spaces to impulsive Volterra integral equations. Then, we give some applications to initial value problems for first order impulsive differential equations in Banach spaces. The results are demonstrated by means of an example of an infinite system for impulsive differential equations.
\end{abstract}

Key words: Impulsive Volterra integral equation, impulsive differential equation, cone and partial ordering, Darbo fixed point theorem.

AMS (MOS) subject classifications: $\quad 45 \mathrm{~N} 05,47 \mathrm{H} 07$.

\section{INTRODUCTION}

Vaughn established an existence theorem of local maximal solutions for nonlinear Volterra integral equations in Banach spaces (see [3] or [2] Theorem 5.5.3). In this paper, we first extend Vaughn's result to nonlinear impulsive Volterra integral equations and obtain existence theorem of global maximal solutions and minimal solutions. And then, we offer some applications to initial value problems for first order impulsive differential equations in Banach spaces. Finally, we give an example of infinite system for impulsive differential equations.

Let the real Banach space $E$ be partially ordered by a cone $P$ of $E$, i.e. $x \leq y$ iff $y-x \in P$. Recall that cone $P$ is said to be solid if its interior $\operatorname{int}(P)$ is not empty. In this case, we write $x \ll y$ iff $y-x \in \operatorname{int}(P)$ (see [1]). Let $J=\left[t_{0}, T\right], t_{0}<t_{1}<\ldots<t_{k}<\ldots<$ $t_{m}<T$ and $P C[J, E]=\{x: x$ is a continuous map from $J$ into $E$ such that $x(t)$ is continuous at $t \neq t_{k}$, left continuous at $t=t_{k}$ and its right limit at $t=t_{k}$ (denoted by $\left.x\left(t_{k}^{+}\right)\right)$exists, $k=1,2, \ldots, m\}$. Evidently, $P C[J, E]$ is a Banach space with norm $\|x\|_{p}=\sup _{t \in J}\|x(t)\|$.

${ }^{1}$ Received: November, 1992. Revised: February, 1993.

${ }^{2}$ Research supported by NNSF-China and Educ. Comm. DF-China. 
Consider the nonlinear impulsive Volterra integral equation in $E$ :

$$
x(t)=x_{0}(t)+\int_{t_{0}}^{t} H(t, s, x(s)) d s+\sum_{t_{0}<t_{k}<t} a_{k}(t) I_{k}\left(x\left(t_{k}\right)\right),
$$

where $\quad x_{0} \in P C[J, E], \quad H \in C[F \times E, E], \quad F=\{(t, s) \in J \times J: t \geq s\}, \quad I_{k} \in C[E, E] \quad$ and $a_{k} \in C\left[J_{k}^{*}, R^{1}\right], J_{k}^{*}=\left[t_{k}, T\right](k=1,2, \ldots, m) . \quad x \in P C[J, E]$ is called a solution of Equation (1) if it satisfies (1) for all $t \in J$.

\section{MAIN THEOREMS}

In the following, let $a_{k}^{*}=\max _{t \in J_{k}^{*}}\left|a_{k}(t)\right|(k=1,2, \ldots, m), T_{r}=\{x \in E:\|x\| \leq r\}$, $B_{r}=\left\{x \in P C[J, E]:\|x\|_{p} \leq r\right\} \quad(r>0), J_{0}=\left[t_{0}, t_{1}\right], \quad J_{1}=\left(t_{1}, t_{2}\right], \ldots, J_{m-1}=\left(t_{m-1}, t_{m}\right]$, $J_{m}=\left(t_{m}, T\right]$ and $\alpha$ denotes the Kuratowski measure of noncompactness (see [2] Section 1.4). For $S \subset P C[J, E]$, we write $S(t)=\{x(t): x \in S\} \subset E(t \in J)$ and $S(J)=\{x(t): x \in S, t \in J\}$ $\subset E$.

Consider the operator $A$ defined by

$$
A x(t)=x_{0}(t)+\int_{t_{0}}^{t} H(t, s, x(s)) d s+\sum_{t_{0}<t_{k}<t} a_{k}(t) I_{k}\left(x\left(t_{k}\right)\right) .
$$

Lemma 1: Suppose that, for any $r>0, H$ is uniformly continuous on $F \times T_{r}, I_{k}$ is bounded on $T_{r}$, and there exists nonnegative constants $L_{r}$ and $M_{r}^{(k)}$ with

$$
2\left(T-t_{0}\right) L_{r}+\sum_{k=1}^{m} a_{k}^{*} M_{r}^{(k)}<1
$$

such that

$$
\begin{gathered}
\alpha(H(t, s, D)) \leq L_{r} \alpha(D),(t, s) \in F, D \subset T_{r}, \\
\alpha\left(I_{k}(D)\right) \leq M_{r}^{(k)} \alpha(D), D \subset T_{r}, k=1,2, \ldots, m .
\end{gathered}
$$

Then, for any $r>0, A$ is a strict set contraction from $B_{r}$ into $P C[J, E]$, i.e. $A$ is continuous and bounded and there exists a constant $0 \leq k_{r}<1$ such that $\alpha(A(S)) \leq k_{r} \alpha(S)$ for any $S \subset B_{r}$.

Proof: It is easy to see that the uniform continuity of $H$ on $F \times T_{r}$ implies the boundedness of $H$ on $F \times T_{r}$, and so $A$ is a bounded and continuous operator from $B_{r}$ into $P C[J, E]$. By the uniform continuity of $H$ and (4) and using Lemma 1.4.1 in [2], we have

$$
\alpha(H(F \times D))=\max _{(t, s) \in F} \alpha(H(t, s, D)) \leq L_{r} \alpha(D), D \subset T_{r}
$$


Now, let $S \subset B_{r}$ be arbitrarily given. By virtue of (2), it is easy to show that the elements of $A(S)$ are equicontinuous on each $J_{k}(k=0,1, \ldots, m)$, and so, by Lemma 1.4.1 in [2],

$$
\alpha(A(S))=\sup _{t \in J} \alpha(A(S(t)))
$$

Using (6), (5) and the obvious formula

$$
\int_{t_{0}}^{t} y(s) d s \in\left(t-t_{0}\right) \overline{c o}\left\{y(s): t_{0}: t_{0} \leq s \leq t\right\}, y \in P C[J, E], t \in J
$$

we find

$$
\begin{aligned}
\alpha(A(S(t))) \leq & \left(t-t_{0}\right) \alpha\left(\overline{c o}\left\{H(t, s, x(s)): x \in S, t_{0} \leq s \leq t\right\}\right) \\
& +\sum_{t_{0}<t_{k}<t} a_{k}^{*} \alpha\left(\left\{I_{k}\left(x\left(t_{k}\right)\right): x \in S\right\}\right) \\
\leq & \left(T-t_{0}\right) \alpha(H(F \times S(J)))+\sum_{k=1}^{m} a_{k}^{*} \alpha\left(I_{k}\left(S\left(t_{k}\right)\right)\right) \\
\leq & \left(T-t_{0}\right) L_{r} \alpha(S(J))+\sum_{k=1}^{m} a_{k}^{*} M_{r}^{(k)} \alpha\left(S\left(t_{k}\right)\right)
\end{aligned}
$$

For any given $\epsilon>0$, there exists a partition $S=\bigcup_{j=1}^{p} S_{j}$ such that

$$
\operatorname{diam}\left(S_{j}\right)<\alpha(S)+\epsilon,(j=1,2, \ldots, p) .
$$

Since $S\left(t_{k}\right)=\bigcup_{j=1}^{p} S_{j}\left(t_{k}\right)$ and $\operatorname{diam}\left(S_{j}\left(t_{k}\right)\right) \leq \operatorname{diam}\left(S_{j}\right)$, we have

$$
\alpha\left(S\left(t_{k}\right)\right) \leq \alpha(S)+\epsilon,(k=1,2, \ldots, m) .
$$

On the other hand, choosing $x_{j} \in S_{j}(j=1,2, \ldots, p)$ and a partition $J_{k}=\bigcup_{i=1}^{n} J_{k}^{(i)}$ $(k=0,1, \ldots, m)$ such that

$$
\left\|x_{j}(t)-x_{j}\left(t^{\prime}\right)\right\|<\epsilon, j=1,2, \ldots, p ; t, t^{\prime} \in J_{k}^{(i)}\left(k=0,1, \ldots, m ; i=1,2, \ldots, n_{k}\right),
$$

we have

$$
S(J)=\bigcup\left\{S_{j}\left(J_{k}^{(i)}\right): i=1,2, \ldots, n_{k} ; k=0,1, \ldots, m ; j=1,2, \ldots, p\right\} .
$$

For $x(t), \bar{x}\left(t^{\prime}\right) \in S_{j}\left(J_{k}^{(i)}\right)$ (i.e. $x, \bar{x} \in S_{j}, t, t^{\prime} \in J_{k}^{(i)}$ ), we find by (10)

$$
\begin{gathered}
\left\|x(t)-\bar{x}\left(t^{\prime}\right)\right\| \leq\left\|x(t)-x_{j}(t)\right\|+\left\|x_{j}(t)-x_{j}\left(t^{\prime}\right)\right\|+\left\|x_{j}\left(t^{\prime}\right)-\bar{x}\left(t^{\prime}\right)\right\| \\
\leq\left\|x-x_{j}\right\|_{p}+\epsilon+\left\|x_{j}-\bar{x}\right\|_{p}<2 \operatorname{diam}\left(S_{j}\right)+\epsilon<2 \alpha(S)+3 \epsilon
\end{gathered}
$$

which implies 


$$
\mathrm{a}(S(J)) \leq 2 a(S)+3 e .
$$

Since e is arbitrary, it follows from (9) and (11) that

$$
\mathrm{a}\left(S\left(t_{k}\right)\right) \leq(S),(k=1,2, \ldots, m)
$$

and

$$
\mathrm{a}(S(J)) \leq 2 a(S)
$$

Finally, (7), (8), (12) and (13) imply a $(A(S)) \leq k_{r} a(S)$, where

$$
k_{r}=2\left(T-t_{0}\right) L_{r}+\sum_{k=1}^{m} a_{k}^{*} M_{r}^{(k)}
$$

By (3), $k_{r}<1$, and the lemma is proved.

Remark 1: The conditions of Lemma 1 are automatically satisfied if $E$ is finite dimensional.

Lemma 2: Let cone $P$ be solid, $H(t, s, x)$ be nondecreasing in $x$ (i.e. $x_{1} \leq x_{2}$ implies $H\left(t, s, x_{1}\right) \leq H\left(t, s, x_{2}\right)$ for $\left.(t, s) \in F\right), I_{k}$ be strongly increasing (i.e. $x_{1} \ll x_{2}$ implies $\left.I_{k}\left(x_{1}\right) \ll I_{k}\left(x_{2}\right), k=1,2, \ldots, m\right), a_{k}(t) \geq 0$ for $t \in J_{k}^{*}$ and $a_{k}\left(t_{k}\right)>0 \quad(k=1,2, \ldots, m)$. If $x_{0}, u, v \in P C[J, E]$ satisfy

and

$$
u(t) \leq x_{0}(t)+\int_{t_{0}}^{t} H(t, s, u(s)) d s+\sum_{t_{0}<t_{k}<t} a_{k}(t) I_{k}\left(u\left(t_{k}\right)\right), t \in J
$$

$$
v(t) \gg x_{0}(t)+\int_{t_{0}}^{t} H(t, s, v(s)) d s+\sum_{t_{0}<t_{k}<t} a_{k}(t) I_{k}\left(v\left(t_{k}\right)\right), t \in J,
$$

then $u\left(t_{0}\right) \ll v\left(t_{0}\right)$ implies $u(t) \ll v(t)$ for $t \in J$.

Proof: Suppose that the conclusion of the lemma is not true. Then the set $Z=\{t \in J: u(t) \ll v(t)$ does not hold $\}$ is not empty. Let $t^{*}=i n f Z$. From the continuity of $u, v$ at $t_{0}$ and $u\left(t_{0}\right) \ll v\left(t_{0}\right)$ we know that $t_{0}<t^{*} \leq T$. So $u(t) \ll v(t)$ for $t_{0} \leq t<t^{*}$. It follows from the left continuous property of $u, v$ at $t=t^{*}$ that $u\left(t^{*}\right) \leq v\left(t^{*}\right)$. Hence, by virtue of (14), (15) and the nondecreasing property of $H$ and strongly increasing property of $I_{k}$,

$$
\begin{gathered}
u\left(t^{*}\right) \leq x_{0}\left(t^{*}\right)+\int_{t_{0}}^{t^{*}} H\left(t^{*}, s, u(s)\right) d s+\sum_{t_{0}<t_{k}<t^{*}} a_{k}^{*}\left(t^{*}\right) I_{k}\left(u\left(t_{k}\right)\right) \\
\leq x_{0}\left(t^{*}\right)+\int_{t_{0}}^{t^{*}} H\left(t^{*}, s, v(s)\right) d s+\sum_{t_{0} \leq t_{k}<t^{*}} a_{k}\left(t^{*}\right) I_{k}\left(v\left(t_{k}\right)\right) \ll v\left(t^{*}\right) .
\end{gathered}
$$


There are two cases:

(a) $\quad t^{*} \neq t_{k}(k=1,2, \ldots, m)$. In this case, $u$ and $v$ are continuous at $t=t^{*}$, and so (16) implies that there exists $\delta>0$ such that $u(t) \ll v(t)$ for $t^{*}<t<t^{*}+\delta$, which contradicts the definition of $t^{*}$.

(b) $t^{*}=t_{k}$ for some $k$. In this case, we have by (14) and (15),

and

$$
u\left(t_{k}^{+}\right) \leq x_{0}\left(t_{k}^{+}\right)+\int_{t_{0}}^{t_{k}} H\left(t_{k}, s, u(s)\right) d s+\sum_{j=1}^{k} a_{j}\left(t_{k}\right) I_{j}\left(u\left(t_{j}\right)\right)
$$

$$
v\left(t_{k}^{+}\right) \geq x_{0}\left(t_{k}^{+}\right)+\int_{t_{0}}^{t_{k}} H\left(t_{k}, s, v(s)\right) d s+\sum_{j=1}^{k} a_{j}\left(t_{k}\right) I_{j}\left(v\left(t_{j}\right)\right),
$$

where $u\left(t_{k}^{+}\right), v\left(t_{k}^{+}\right), x_{0}\left(t_{k}^{+}\right)$denote the right limits of $u, v, x_{0}$ at $t=t_{k}$, respectively. It follows from (16), (17), (18) and the strongly increasing property of $I_{k}$ that $u\left(t_{k}^{+}\right) \ll v\left(t_{k}^{+}\right)$, and therefore, there exists $\delta^{*}>0$ such that $u(t) \ll v(t)$ for $t^{*}=t_{k}<t<t_{k}+\delta^{*}$, which contradicts the definition of $t^{*}$ too.

The proof is complete.

Remark 2: Lemma 2 is also true if (14) and (15) are replaced by

and

$$
u(t) \ll x_{0}(t)+\int_{t_{0}}^{t} H(t, s, u(s)) d s+\sum_{t_{0}<t_{k}<t} a_{k}(t) I_{k}\left(u\left(t_{k}\right)\right), t \in J
$$

$$
v(t) \geq x_{0}(t)+\int_{t_{0}}^{t} H(t, s, v(s)) d s+\sum_{t_{0}<t_{k}<t} a_{k}(t) I_{k}\left(v\left(t_{k}\right)\right), t \in J .
$$

Theorem 1: $\quad$ Let the conditions of Lemma 1 be satisfied. Suppose that

$$
\left(T-t_{0}\right) c+\sum_{k=1}^{m} a_{k}^{*} c_{k}<1
$$

where

$$
c=\varlimsup_{\|x\| \rightarrow \infty}\left(\sup _{(t, s) \in F} \frac{\|H(t, s, x)\|}{\|x\|}\right)
$$

and

$$
c_{k}=\overline{\lim }_{\|x\| \rightarrow \infty} \frac{\left\|I_{k}(x)\right\|}{\|x\|}(k=1,2, \ldots, m) .
$$

Then, Equation (1) has a solution in $P C[J, E]$. 
Proof: $\quad$ Choose $c^{\prime}>c$ and $c_{k}^{\prime}>c_{k}(k=1,2, \ldots, m)$ such that

$$
b=\left(T-t_{0}\right) c^{\prime}+\sum_{k=1}^{m} a_{k}^{*} c_{k}^{\prime}<1 .
$$

By virtue of (20) and (21), there exists $r>0$ such that

$$
\|H(t, s, x)\|<c^{\prime}\|x\|,\|x\| \geq r,(t, s) \in F
$$

and

$$
\left\|I_{k}(x)\right\|<c_{k}^{\prime}\|x\|,\|x\| \geq r(k=1,2, \ldots, m)
$$

so

$$
\|H(t, s, x)\| \leq c^{\prime}\|x\|+N, x \in E,(t, s) \in F
$$

and

$$
\left\|I_{k}(x)\right\| \leq c_{k}^{\prime}\|x\|+N, x \in E
$$

where

$$
N=\max \left\{\sup _{(t, s) \in F, x \in T_{r}}\|H(t, s, x)\|, \sup _{x \in T_{r}}\left\|I_{k}(x)\right\|, k=1,2, \ldots, m\right\}
$$

Let

$$
R=N^{\prime}(1-b)^{-1}
$$

where $b$ is defined by $(22)$ and $N^{\prime}=\left\|x_{0}\right\|_{p}+\left(T-t_{0}+\sum_{k=1}^{m} a_{k}^{*}\right) N$. For any $x \in B_{R}$ (i.e. $\left.\|x\|_{p} \leq R\right)$, we have by (2), (23), and (24),

$$
\|A x(t)\| \leq\left\|x_{0}(t)\right\|+\int_{t_{0}}^{t}\left(c^{\prime}\|x(s)\|+N\right) d s+\sum_{k=}^{m} a_{k}^{*}\left(c_{k}^{\prime}\left\|x\left(t_{k}\right)\right\|+N\right), t \in J
$$

and so

$$
\begin{gathered}
\|A x\|_{p} \leq\left\|x_{0}\right\|_{p}+\left(T-t_{0}\right)\left(c^{\prime}\|x\|_{p}+N\right)+\sum_{k=1}^{m} a_{k}^{*}\left(c_{k}^{\prime}\|x\|_{p}+N\right) \\
=b\|x\|_{p}+N^{\prime} \leq b R+N^{\prime}=R .
\end{gathered}
$$

Consequently, $A: B_{R} \rightarrow B_{R}$. On the other hand, by Lemma 1, $A$ is a strict set contraction. Hence, the Darbo fixed point theorem (see [2] theorem 5.3.1) implies that $A$ has a fixed point in $B_{R}$. The proof is complete.

Theorem 2: $\quad$ Let the assumptions of Theorem 1 hold. Suppose that cone $P$ is solid, $H(t, s, x)$ is nondecreasing in $x$ and $I_{k}$ are strongly increasing $(k=1,2, \ldots, m)$. Then Equation (1) has maximal solution $v$ and minimal solution $u$ in $P C[J, E]$, i.e. 
$u(t) \leq x(t) \leq v(t)(t \in J)$ for any solution $x$ of Equation (1) in $P C[J, E]$.

Proof: $\quad$ Choose $y \in \operatorname{int}(P),\|y\|=1$ and let

$$
\begin{gathered}
A_{n} x(t)=A x(t)+\frac{1}{n} y \\
=x_{0}(t)+\frac{1}{n} y+\int_{t_{0}}^{t} H(t, s, x(s)) d s+\sum_{t_{0}<t_{k}<t} a_{k}(t) I_{k}\left(x\left(t_{k}\right)\right),(n=1,2,3, \ldots) .
\end{gathered}
$$

By Theorem 1 and (25), $A_{n}$ has a fixed point $x_{n}$ in $P C[J, E]$ such that $\left\|x_{n}\right\|_{p} \leq R_{n}=$ $N_{n}^{\prime}(1-b)^{-1}$, where $N_{n}^{\prime}=\left\|x_{0}+n^{-1} y\right\|_{p}+\left(T-t_{0}+\sum_{k=1}^{m} a_{k}^{*}\right) N$. So,

$$
\left\|x_{n}\right\|_{p} \leq R^{*},(n=1,2,3, \ldots)
$$

where

$$
R^{*}=(1-b)^{-1}\left\{\left\|x_{0}\right\|_{p}+\|y\|+\left(T-t_{0}+\sum_{k=1}^{m} a_{k}^{*}\right) N\right\}=\text { const }
$$

and

$$
\begin{gathered}
x_{n}(t)=A x_{n}(t)+\frac{1}{n} y \\
=x_{0}(t)+\frac{1}{n} y+\int_{t_{0}}^{t} H\left(t, s, x_{n}(s)\right) d s+\sum_{t_{0}<t_{k}<t} a_{k}(t) I_{k}\left(x_{n}\left(t_{k}\right)\right),(n=1,2,3, \ldots) .
\end{gathered}
$$

Let $S=\left\{x_{n}: n=1,2,3, \ldots\right\}$. By virtue of $(27)$ and Lemma 1 , there exists $0 \leq k^{*}<1$ such that $\alpha(A(S)) \leq k^{*} \alpha(S)$. On the other hand, (28) implies

$$
\alpha(S) \leq \alpha(A(S))+\alpha\left(\left\{\frac{1}{n} y: n=1,2,3, \ldots\right\}\right)=\alpha(A(S)) .
$$

So, we have $\alpha(S)=0$, and hence, there are $\left\{x_{n_{i}}\right\} \subset\left\{x_{n}\right\}$ and $v \in P C[J, E\}$ such that $\left\|x_{n_{i}}-v\right\|_{p} \rightarrow 0(i \rightarrow \infty)$. Observing the uniform continuity of $H$ on $F \times T_{R^{*}}$ and taking limit in (28) along $n_{i}$, we get

$$
v(t)=x_{0}(t)+\int_{t_{0}}^{t} H(t, s, v(s)) d s+\sum_{t_{0}<t_{k}<t} a_{k}(t) I_{k}\left(v\left(t_{k}\right)\right), t \in J
$$

i.e. $v$ is a solution of Equation (1).

Now, let $x$ be any solution of Equation (1) in $P C[J, E]$, i.e.

By (28), we have

$$
x(t)=x_{0}(t)+\int_{t_{0}}^{t} H(t, s, x(s)) d s+\sum_{t_{0}<t_{k}<t} a_{k}(t) I_{k}\left(x\left(t_{k}\right)\right), t \in J .
$$

$$
x_{n}(t) \gg x_{0}(t)+\int_{t_{0}}^{t} H\left(t, s, x_{n}(s)\right) d s+\sum_{t_{0}<t_{k}<t} a_{k}(t) I_{k}\left(x_{n}\left(t_{k}\right)\right) t \in J
$$


In addition,

$$
x_{n}\left(t_{0}\right)=x_{0}\left(t_{0}\right)+\frac{1}{n} y \gg x_{0}\left(t_{0}\right)=x\left(t_{0}\right)
$$

So, (29), (30), (31) and Lemma 2 imply

$$
x_{n}(t) \gg x(t), t \in J, n=1,2,3, \ldots .
$$

Taking the limit in (32) along $n_{i}$, we obtain $v(t) \geq x(t)$ for $t \in J$. Consequently, $v$ is the maximal solution of Equation (1) in $P C[J, E]$.

Similarly, considering sequence of operators

$$
\begin{gathered}
A_{n}^{*} x(t)=A x(t)-\frac{1}{n} y \\
=x_{0}(t)-\frac{1}{n} y+\int_{t_{0}}^{t} H(t, s, x(s)) d s+\sum_{t_{0}<t_{k}<t} a_{k}(t) I_{k}\left(x\left(t_{k}\right)\right) \quad(n=1,2,3, \ldots)
\end{gathered}
$$

instead of sequence (26), we can get the minimal solution $u$ of Equation (1) in $P C[J, E]$. The proof is complete.

Theorem 3: $\quad$ Let the assumptions of Theorem 2 hold. Let $m \in P C[J, E]$ and $u$ and $v$ be the minimal and maximal solutions of Equation (1) in $P C[J, E]$ respectively. The following conclusions hold:

(a) if

$$
m(t) \leq x_{0}(t)+\int_{t_{0}}^{t} H(t, s, m(s)) d s+\sum_{t_{0}<t_{k}<t} a_{k}(t) I_{k}\left(m\left(t_{k}\right)\right), t \in J
$$

then $m(t) \leq v(t)$ for $t \in J$.

(b) if

$$
m(t) \geq x_{0}(t)+\int_{t_{0}}^{t} H(t, s, m(s)) d s+\sum_{t_{0}<t_{k}<t} a_{k}(t) I_{k}\left(m\left(t_{k}\right)\right), t \in J
$$

then $m(t) \geq u(t)$ for $u \in J$.

Proof: We need only to prove $(a)$ since the proof of $(b)$ is similar. As in the proof of Theorem 2, (30) holds, and, by (33),

$$
x_{n}\left(t_{0}\right)=x_{0}\left(t_{0}\right)+\frac{1}{n} y \gg x_{0}\left(t_{0}\right) \geq m\left(t_{0}\right) .
$$

It follows from (30), (33), (34) and Lemma 2 that 


$$
x_{n}(t) \gg m(t), t \in J, n=1,2,3, \ldots
$$

which implies by taking limit along $n_{i}$ that $v(t) \geq m(t)$ for $t \in J$. The proof is complete.

\section{APPLICATIONS}

This section applies Theorem 2 to the IVP of the nonlinear impulsive differential equation in $E$ :

$$
\left\{\begin{array}{cc}
x^{\prime}=f(t, x), t \neq t_{k} & (k=1,2, \ldots, m), \\
\left.\Delta x\right|_{t=t_{k}}=I_{k}\left(x\left(t_{k}\right)\right), & (k=1,2, \ldots, m), \\
x(0)=x_{0}, &
\end{array}\right.
$$

where $f \in C[J \times E, E], J=[0, T](T>0), x_{0} \in E, I_{k} \in C[E, E], 0<t_{1}<\ldots<t_{k}<\ldots<t_{m}$ $<T$. Let $J^{\prime}=J \backslash\left\{t_{1}, \ldots, t_{m}\right\} . \quad x \in P C[J, E] \cap C^{1}\left[J^{\prime}, E\right]$ is called a solution of IVP (35) if it satisfies (35).

Lemma 3: Let $y, z \in P C[J, E]$ and $M$ be a constant. Then, the IVP of linear impulsive differential equation

$$
\left\{\begin{array}{cl}
x^{\prime}+M x=y(t), t \neq t_{k} & (k=1,2, \ldots, m), \\
\left.\Delta x\right|_{t=t_{k}}=I_{k}\left(z\left(t_{k}\right)\right), & (k=1,2, \ldots, m), \\
x(0)=x_{0} &
\end{array}\right.
$$

has a unique solution in $P C[J, E] \cap C^{1}[J, E]$ which is given by

$$
x(t)=x_{0} e^{-M t}+\int_{0}^{t} e^{-M(t-s)} y(s) d s+\sum_{0<t_{k}<t} e^{-M\left(t-t_{k}\right)} I_{k}\left(z\left(t_{k}\right)\right) .
$$

Proof: Let $x(t)$ be defined by (37). Evidently, $x \in P C[J, E], x(0)=x_{0}$ and $\left.\Delta x\right|_{t=t_{k}}=I_{k}\left(z\left(t_{k}\right)\right)$. By (37), we have

$$
x(t) e^{M t}=x_{0}+\int_{0}^{t} e^{M s} y(s) d s+\sum_{0<t_{k}<t} e^{M} t_{k} I_{k}\left(z\left(t_{k}\right)\right)
$$

so, direct differentiation implies, for $t \neq t_{k}$,

$$
\left(x^{\prime}(t)+M x(t)\right) e^{M t}=e^{M t} y(t) .
$$

Hence, $x \in C^{1}\left[J^{\prime}, E\right]$ and it is a solution of (36).

It remains to show the uniqueness of solution. Let $x_{1}, x_{2} \in P C[J, E] \cap C^{1}\left[J^{\prime}, E\right]$ be 
two solutions of (36) and let $\bar{x}=x_{1}-x_{2}$. Then $\bar{x}^{\prime}+M \bar{x}=\theta$ for $t \neq t_{k}$, where $\theta$ denotes the zero element of $E$, and so

$$
\left(\bar{x} e^{M t}\right)^{\prime}=\left(\bar{x}^{\prime}+M \bar{x}\right) e^{M t}=\theta, t \neq t_{k}(k=1,2, \ldots, m) .
$$

Since $\bar{x}(0)=\theta$, it follows that

$$
\bar{x}(t)=\bar{x}(0) e^{-M t}=\theta \text { for } 0 \leq t \leq t_{1} .
$$

On the other hand,

$$
\left.\Delta x_{1}\right|_{t=t_{1}}=I_{1}\left(z\left(t_{1}\right)\right)=\left.\Delta x_{2}\right|_{t=t_{1}}
$$

hence

$$
x_{1}\left(t_{1}^{+}\right)=x_{1}\left(t_{1}\right)+\left.\Delta x_{1}\right|_{t=t_{1}}=x_{2}\left(t_{1}\right)+\Delta x_{2} \mid t_{t=t_{1}}=x_{2}\left(t_{1}^{+}\right) .
$$

It follows from (38) and (39) that

$$
\bar{x}(t)=\bar{x}\left(t_{1}^{+}\right) e^{-M\left(t-t_{1}\right)}=\theta \text { for } t_{1}<t \leq t_{2} .
$$

Similarly, we can show $\bar{x}(t)=\theta$ for $t_{2}<t \leq T$, i.e. $x_{1}=x_{2}$, and the lemma is proved.

Now, consider the impulsive Volterra integral equation

$$
\begin{gathered}
x(t)=x_{0} e^{-M t}+\int_{0}^{t} e^{-M(t-s)}[f(s, x(s))+M x(s)] d s \\
+\sum_{0<t_{k}<t} e^{-M\left(t-t_{k}\right)} I_{k}\left(x\left(t_{k}\right)\right) .
\end{gathered}
$$

Lemma 4: $x \in P C[J, E] \cap C^{1}\left[J^{\prime}, E\right]$ is a solution of $I V P$ (35) if and only if $x \in P C[J, E]$ is a solution of Equation (40).

Proof: $\quad$ For $z \in P C[J, E]$, Lemma 3 implies that the linear problem

$$
\left\{\begin{array}{cc}
x^{\prime}=f(t, z)-M(x-z), t \neq t_{k} & (k=1,2, \ldots, m), \\
\left.\Delta x\right|_{t=t_{k}}=I_{k}\left(z\left(t_{k}\right)\right), & (k=1,2, \ldots, m), \\
x(0)=x_{0} &
\end{array}\right.
$$

has a unique solution of $P C[J, E] \cap C^{1}\left[J^{\prime}, E\right]$ which is given by

Let $x=B z$, i.e.

$$
\begin{aligned}
x(t)=x_{0} e^{-M t} & +\int_{0}^{t} e^{-M(t-s)}[f(s, z(s))+M z(s)] d s \\
& +\sum_{0 \leq t_{k}<t} e^{-M\left(t-t_{k}\right)} I_{k}\left(z\left(t_{k}\right)\right) .
\end{aligned}
$$




$$
\begin{gathered}
B z(t)=x_{0} e^{-M t}+\int_{0}^{t} e^{-M(t-s)}[f(s, z(s))+M z(s)] d s \\
+\sum_{0<t_{k}<t} e^{-M\left(t-t_{k}\right)} I_{k}\left(z\left(t_{k}\right)\right) .
\end{gathered}
$$

It is easy to see from (41) that $x$ is a solution of IVP (35) if and only if $x=B z=z$, that is, $x$ is a solution of Equation (40). The lemma is proved.

Theorem 4: $\quad$ Let cone $P$ be solid and $I_{k}$ be strongly increasing $(k=1,2, \ldots$,$) .$ Assume that, for any $r>0, f$ is uniformly continuous on $J \times T_{r}$ and there exist nonnegative constants $L_{r}$ and $M_{r}^{(k)}$ with

$$
2 T\left(L_{r}+M\right)+\sum_{k=1}^{m} M_{r}^{(k)}<1
$$

such that

$$
\alpha(f(t, D)) \leq L_{r} \alpha(D), t \in J, D \subset T_{r}
$$

and

$$
\alpha\left(I_{k}(D)\right) \leq M_{r}^{(k)} \alpha(D), D \subset T_{r} \quad(k=1,2, \ldots, m),
$$

where $M$ is a nonnegative constant independent of $r$. Assume further that,

$$
T(c+M)+\sum_{k=1}^{m} c_{k}<1
$$

where

$$
c=\varlimsup_{\|x\| \rightarrow \infty}\left(\sup _{t \in J} \frac{\|f(t, x)\|}{\|x\|}\right), c_{k}=\varlimsup_{\|x\| \rightarrow \infty} \frac{\left\|I_{k}(x)\right\|}{\|x\|}(k=1,2, \ldots, m),
$$

and

$$
f(t, x)-f(t, y) \geq-M(x-y) \text { for } x \geq y \quad(x, y \in E) .
$$

Then IVP (35) has maximal solution $v$ and minimal solution $u$ in $P C[J, E] \cap C^{1}\left[J^{\prime}, E\right]$, i.e. $u(t) \leq x(t) \leq v(t)(t \in J)$ for any solution $x$ of IVP (35) in $P C[J, E] \cap C^{1}\left[J^{\prime}, E\right]$.

Proof: By Lemma 4, $x \in P C[J, E] \cap C^{1}\left[J^{\prime}, E\right]$ is a solution of IVP (35) iff $x \in P C[J, E]$ is a solution of Equation (40). Evidently, (40) is an integral equation of type (1), where $t_{0}=0$,

$$
\begin{gathered}
x_{0}(t)=x_{0} e^{-M t}, H(t, s, x)=e^{-M(t-s)}[f(s, x)+M x] \\
a_{k}(t)=e^{-M\left(t-t_{k}\right)}(k=1,2, \ldots, m) .
\end{gathered}
$$

Since

$$
\alpha(H(t, s, D)) \leq \alpha(f(s, D))+M \alpha(D) \leq\left(L_{r}+M\right) \alpha(D),(t, s) \in F, D \subset T_{r},
$$




$$
\begin{gathered}
a_{k}(t)>0 \text { for } t \in I_{k}, a_{k}\left(t_{k}\right)=a_{k}^{*}=1, \\
\varlimsup_{\|x\| \rightarrow \infty}\left(\sup _{(t, s) \in F} \frac{\|H(t, s, x)\|}{\|x\|}\right) \leq \varlimsup_{\|x\| \rightarrow \infty}\left(\sup _{s \in J} \frac{\|f(s, x)\|+M\|x\|}{\|x\|}\right)=c+M,
\end{gathered}
$$

and, by virtue of $(47), H(t, s, x)$ is nondecreasing in $x$, all conditions of Theorem 2 are satisfied, and so, Theorem 2 implies that Equation (40) has maximal solution $v$ and minimal solution $u$ in $P C[J, E]$. The proof is complete.

Example: Consider the IVP in terms of the infinite system of impulsive differential equations:

$$
\begin{gathered}
x_{n}^{\prime}=\frac{1}{n+3}\left(2 t-x_{n}\right)=\sqrt[3]{\frac{t^{2}-x_{n+1}}{n}}+\frac{1}{8 n^{2}} \ln \left(1+e^{t} x_{2 n}\right), 0 \leq t \leq 1, t \neq \frac{1}{2} \\
\left.\Delta x_{n}\right|_{t=\frac{1}{2}}=\frac{1}{4} x_{n}\left(\frac{1}{2}\right) \\
x_{n}(0)=\cos n,(n=1,2,3, \ldots) .
\end{gathered}
$$

A solution $x=\left(x_{1}, \ldots, x_{n}, \ldots\right)$ of IVP (48) is called bounded if $\operatorname{sun}_{n} p\left|x_{n}(t)\right|<\infty$ for $0 \leq t \leq 1$.

Conclusion: IVP (48) has maximal bounded solution $v=\left(v_{1}, \ldots, v_{n}, \ldots\right)$ and minimal bounded solution $u=\left(u_{1}, \ldots, u_{n}, \ldots\right)$, i.e. for any bounded solution $x=\left(x_{1}, \ldots, x_{n}, \ldots\right)$ of $(48)$, $u(t) \leq x(t) \leq v(t)(t \in[0,1])$ holds.

Proof: Let $E=\left\{x=\left(x_{1}, \ldots, x_{n}, \ldots\right): s \sup _{n}\left|x_{n}\right|<\infty\right\}$ with norm $\|x\|=\sup _{n} p$ $\left|x_{n}\right|, P=\left\{x=\left(x_{1}, \ldots, x_{n}, \ldots\right\} \in E ; x_{n} \geq 0, n=1,2,3, \ldots\right\}$. It is well known that $E$ is a Banach space and $P$ is a solid cone in $E$ with $\operatorname{int}(P)=\left\{x=\left(x_{1}, \ldots, x_{n}, \ldots\right) \in E ;\right.$ inf $\left.x_{n}>0\right\}$. Evidently, the bounded solution $x=\left(x_{1}, \ldots, x_{n}, \ldots\right)$ of IVP $(48)$ is equivalent to the solution $x \in P C[J, E] \cap C^{1}\left[J^{\prime}, E\right]$ of the following IVP in $E$ :

$$
\left\{\begin{array}{c}
x^{\prime}=f(t, x), \\
\left.\Delta x\right|_{t=\frac{1}{2}}=\frac{1}{4} x\left(\frac{1}{2}\right), \\
x(0)=x_{0},
\end{array}\right.
$$

where $x_{0}=(\cos 1, \ldots, \cos n, \ldots) \in E, f=\left(f_{1}, \ldots, f_{n}, \ldots\right)$, in which

$$
f_{n}(t, x)=\frac{1}{n+3}\left(2 t-x_{n}\right)-\sqrt[3]{\frac{t^{2}-x_{n+1}}{n}}+\frac{1}{8 n^{2}} \ln \left(1+e^{t} x_{2 n}\right),(n=1,2,3, \ldots),
$$

$T=1, J=[0,1], m=1, t_{1}=\frac{1}{2}$ and

$$
I_{1}(x)=\frac{1}{4} x
$$

Obviously, (49) is of the form (35). It is clear that $f \in C[J \times E, E]$ and, for any $r>0, f$ is 
uniformly continuous on $J \times T_{r}, I_{1} \in C[E, E], I_{1}$ is strongly increasing and

$$
\alpha\left(I_{1}(D)\right)=\frac{1}{4} \alpha(D) \text { for bounded } D \subset E
$$

We now show

$$
\alpha(f(t, D))=0 \text { for } t \in J \text { and bounded } D \subset E .
$$

In fact, let $x^{(p)}=\left(x_{1}^{(p)}, \ldots, x_{n}^{(p)}, \ldots\right) \in D \quad$ and $\quad y^{(p)}=f\left(t, x^{(p)}\right), y^{(p)}=\left(y_{1}^{(p)}, \ldots, y_{n}^{(p)}, \ldots\right)$ $(p=1,2,3, \ldots)$. Then there exists positive constant $d$ such that

$$
\left|x_{n}^{(p)}\right| \leq d n,(p=1,2,3, \ldots)
$$

Since, by (50),

$$
y_{n}^{(p)}=f_{n}\left(t, x^{(p)}\right)=\frac{1}{n+3}\left(2 t-x_{n}^{(p)}\right)-3 \sqrt{\frac{t^{2}-x_{n+1}^{(p)}}{n}}+\frac{1}{8 n^{2}} \ln \left(1+e^{t} x_{2 n}^{(p)}\right)
$$

we have

$$
\left|y_{n}^{(p)}\right| \leq \frac{2+d}{n+3}+\sqrt[3]{\frac{1+d}{n}}+\frac{1}{8 n^{2}} \ln \left(1+e^{d}\right),(n, p=1,2,3, \ldots) .
$$

Hence $\left\{y_{n}^{(p)}\right\}$ is bounded, so, by the diagonal method, we can select a subsequence $\left\{p_{i}\right\}$ of $\{p\}$ such that

$$
\lim _{i \rightarrow \infty} y_{n}^{\left(p_{i}\right)}=y_{n} \quad(n=1,2,3, \ldots)
$$

It follows from (54) that

$$
\left|y_{n}\right| \leq \frac{2+d}{n+3}+\sqrt[3]{\frac{1+d}{n}}+\frac{1}{8 n^{2}} \ln \left(1+e^{d}\right), \quad(n=1,2,3, \ldots) .
$$

Consequently, $y=\left(y_{1}, \ldots, y_{n}, \ldots\right) \in E$. For any given $\epsilon>0,(54)$ and (56) imply that there exists a positive integer $N$ such that

$$
\left|y_{n}^{\left(p_{i}\right)}\right|<\frac{\epsilon}{3},\left|y_{n}\right|<\frac{\epsilon}{3} \text { for } n>N, i=1,2,3, \ldots .
$$

On the other hand, (55) implies that there exists $i_{0}$ such that

$$
\left|y_{n}^{\left(p_{i}\right)}-y_{n}\right|<\frac{\epsilon}{3} \text { for } i>i_{0}, n=1,2, \ldots, N \text {. }
$$

It follows from (57) and (58) that

$$
\left\|y^{\left(p_{i}\right)}-y\right\|=\sup _{n}\left|y_{n}^{\left(p_{i}\right)}-y_{n}\right|<\epsilon \text { for } i>i_{0}
$$

i.e. $\left\|y^{\left(p_{i}\right)}-y\right\| \rightarrow 0$. Hence, $f(t, D)$ is relatively compact and (53) holds. For $x=\left(x_{1}, \ldots, x_{n}, \ldots\right) \in E, y=\left(y_{1}, \ldots, y_{n}, \ldots\right) \in E, x \geq y$ (i.e. $\left.x_{n} \geq y_{n}, n=1,2,3, \ldots\right)$ and $t \in J$, we have by $(50)$, 


$$
\begin{aligned}
f_{n}(t, x)-f_{n}(t, y)= & -\frac{1}{n+3}\left(x_{n}-y_{n}\right)+\frac{1}{\sqrt[3]{n}}\left(\sqrt[3]{x_{n+1}-t^{2}}-\sqrt[3]{y_{n+1}-t^{2}}\right) \\
& +\frac{1}{8 n^{2}}\left(\ln \left(1+e^{t} x_{2 n}\right)-\ln \left(1+e^{t} y_{2 n}\right)\right) \\
& \geq-\frac{1}{4}\left(x_{n}-y_{n}\right), n=1,2,3, \ldots,
\end{aligned}
$$

so, (47) is satisfied for $M=\frac{1}{4}$ and (43) is also satisfied since, by (52) and (53), $L_{r}=0$, $M_{r}^{(1)}=\frac{1}{4}$ and $2 T\left(L_{r}+M\right)+M_{r}^{(1)}=\frac{1}{2}+\frac{1}{4}<1$.

Now, we check (46). By virtue of $(50)$, we have

$$
\|f(t, x)\| \leq \frac{1}{4}(2 t+\|x\|)+\sqrt[3]{t^{2}+\|x\|}+\frac{1}{8} \ln \left(1+e^{t\|x\|}\right),
$$

and so

$$
\begin{gathered}
c=\overline{\lim }_{\|x\| \rightarrow \infty}\left(\sup _{t \in J} \frac{\|f(t, x)\|}{\|x\|}\right) \\
\leq \lim _{\|x\| \rightarrow \infty}\|x\|^{-1}\left(\frac{1}{4}(2+\|x\|)+\sqrt[3]{1+\|x\|}+\frac{1}{8} \ln \left(1+e^{\|x\|}\right)\right)=\frac{3}{8} .
\end{gathered}
$$

On the other hand, (51) implies

$$
c_{1}=\varlimsup_{\|x\| \rightarrow \infty} \frac{\left\|I_{1}(x)\right\|}{\|x\|}=\frac{1}{4} .
$$

Hence $T(c+M)+c_{1} \leq \frac{7}{8}<1$, i.e. (46) holds. Finally, our conclusion follows from Theorem 4 .

\section{REFERENCES}

[1] Guo, D. and Lakshmikantham, V., Nonlinear Problems in Abstract Cones, Academic Press, Inc., Boston and New York 1988.

[2] Lakshmikantham, V. and Leela, S., Nonlinear Differential Equations in Abstract Spaces, Pergamon Press, Oxford 1981.

[3] Vaughn, R., Existence and comparison results for nonlinear Volterra integral equations in a Banach space, Appl. Anal. 7 (1978), 337-348. 


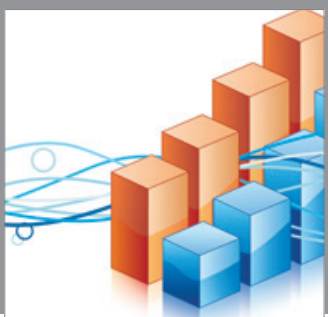

Advances in

Operations Research

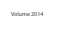

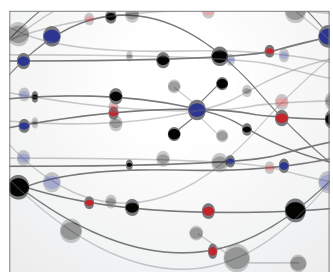

\section{The Scientific} World Journal
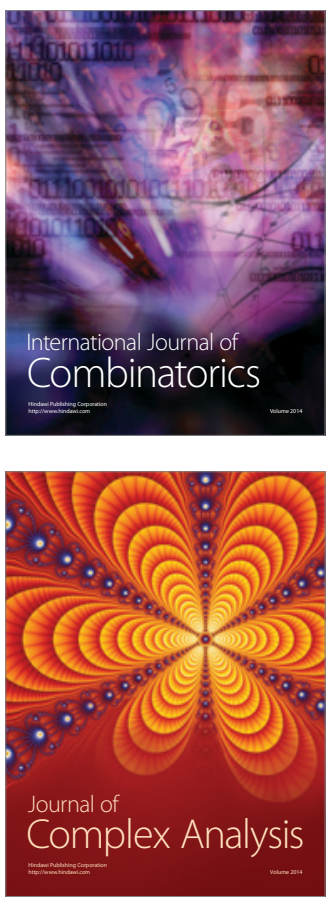

International Journal of

Mathematics and

Mathematical

Sciences
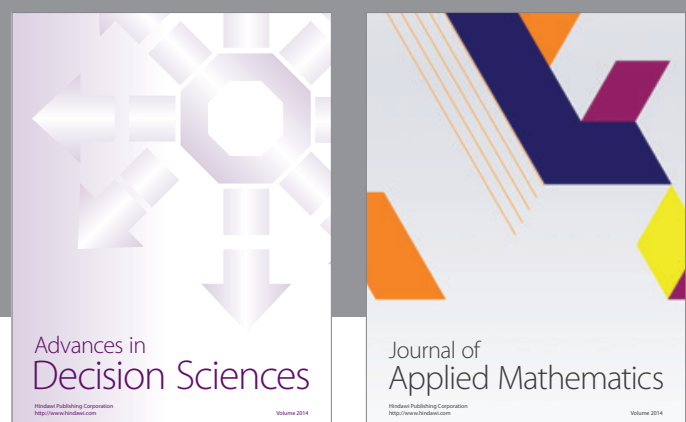

Journal of

Applied Mathematics
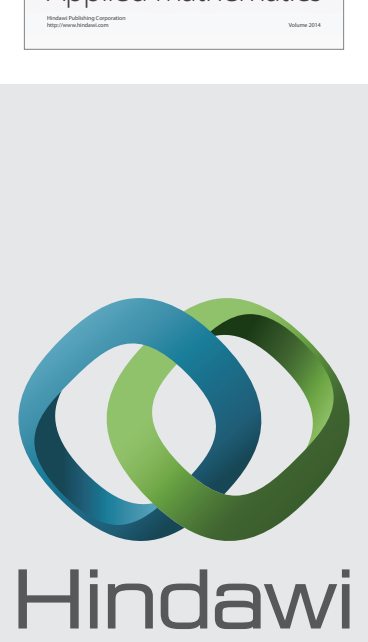

Submit your manuscripts at http://www.hindawi.com
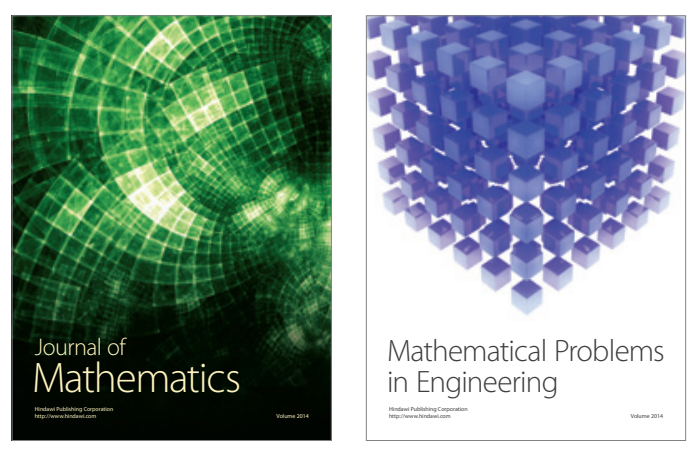

Mathematical Problems in Engineering
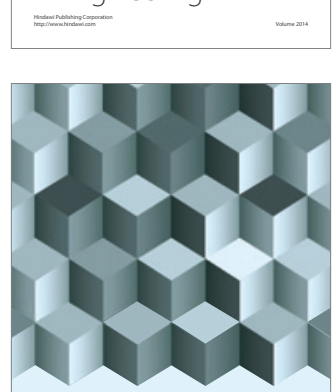

Journal of

Function Spaces
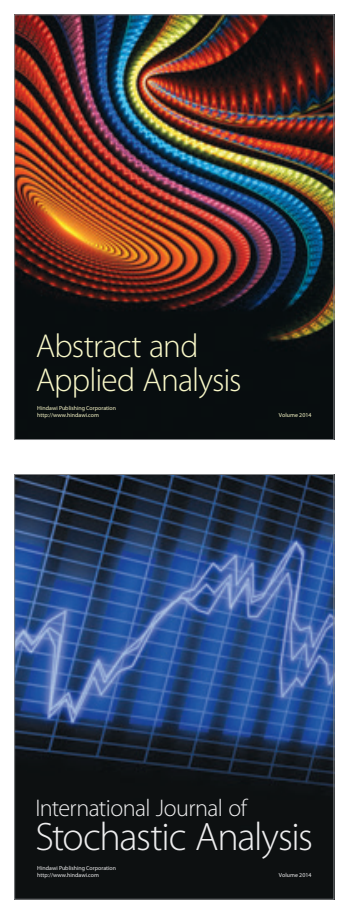

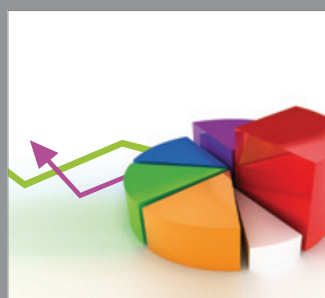

ournal of

Probability and Statistics

Promensencen
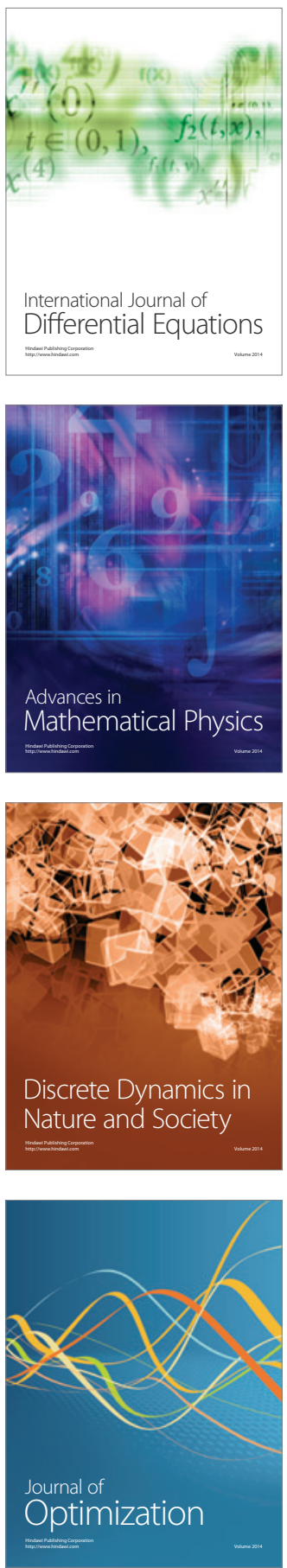